\title{
Cost-effectiveness of health-related lifestyle advice delivered by peer or lay advisors: synthesis of evidence from a systematic review
}

Mark Pennington ${ }^{1}$, Shelina Visram ${ }^{2}$, Cam Donaldson $^{3}$, Martin White $^{4}$, Monique Lhussier $^{5}$, Katherine Deane ${ }^{6}$, Natalie Forster ${ }^{5}$ and Susan M Carr ${ }^{5^{*}}$

\begin{abstract}
Background: Development of new peer or lay health-related lifestyle advisor (HRLA) roles is one response to the need to enhance public engagement in, and improve cost-effectiveness of, health improvement interventions. This article synthesises evidence on the cost-effectiveness of HRLA interventions aimed at adults in developed countries, derived from the first systematic review of the effectiveness, cost-effectiveness, equity and acceptability of different types of HRLA role.

Methods: The best available evidence on the cost-effectiveness of HRLA interventions was obtained using systematic searches of 20 electronic databases and key journals, as well as searches of the grey literature and the internet. Interventions were classified according to the primary health behaviour targeted and intervention costs were estimated where necessary. Lifetime health gains were estimated (in quality-adjusted life years, where possible), based on evidence of effectiveness of HRLAs in combination with published estimates of the lifetime health gains resulting from lifestyle changes, and assumptions over relapse. Incremental cost-effectiveness ratios are reported.
\end{abstract}

Results: Evidence of the cost-effectiveness of HRLAs was identified from 24 trials included in the systematic review. The interventions were grouped into eight areas. We found little evidence of effectiveness of HRLAs for promotion of exercise/improved diets. Where HRLAs were effective cost-effectiveness varied considerably: Incremental Cost effectiveness Ratios were estimated at $£ 6,000$ for smoking cessation; $£ 14,000$ for a telephone based type 2 diabetes management; and $£ 250,000$ or greater for promotion of mammography attendance and for HIV prevention amongst drug users. We lacked sufficient evidence to estimate ICERs for breastfeeding promotion and mental health promotion, or to assess the impact of HRLAs on health inequalities.

Conclusions: Overall, there is limited evidence suggesting that HRLAs are cost-effective in terms of changing health-related knowledge, behaviours or health outcomes. The evidence that does exist indicates that HRLAs are only cost-effective when they target behaviours likely to have a large impact on overall health-related quality of life. Further development of HRLA interventions needs to target specific population health needs where potential exists for significant improvement, and include rigorous evaluation to ensure that HRLAs provide sufficient value for money.

Keywords: Health-related lifestyle advisor, Peer counsellor, Cost-effectiveness

\footnotetext{
* Correspondence: sue.carr@northumbria.ac.uk

${ }^{5}$ Faculty of Health \& Life Sciences/Fuse UKCRC Centre for Translational Research in Public Health, Room H012, Coach Lane Campus East, Northumbria University, Newcastle-upon-Tyne NE7 7XA, UK

Full list of author information is available at the end of the article
} 


\section{Introduction}

Behaviour is recognised as a key determinant of health, with modifiable lifestyle factors such as smoking, physical inactivity, poor diet and alcohol consumption, contributing significantly to morbidity and mortality [1]. This is particularly the case in developed countries, where these major health risks tend to be more prevalent amongst the lower socio-economic groups and therefore contribute to social inequalities in health [2,3]. Evidence from the UK indicates that the decline in the proportion of the population that engages in unhealthy behaviours has been mainly in higher socio-economic groups resulting in widening inequalities [4]. The UK government has responded with public health policy setting out the action needed to encourage and enable individuals to make healthier lifestyle choices, with a particular focus on those living in socioeconomically disadvantaged areas [5].

Awareness of the role of behavioural factors in health has increased in the general population in developed countries, along with a growing chronic disease burden [6]. Healthcare systems have responded with a shift from paternalistic to partnership models of care, and sought to promote increased involvement of patients and the wider public [7]. The expansion of roles to deliver health-related lifestyle advice represents one strategy in this new partnership approach. An example from the UK is the National Health Service (NHS) health trainer role, which aims to empower people to adopt healthy behaviours by offering practical support from someone who understands the pressures they face [5]. Similar types of lay or peer-based health-related lifestyle advisor (HRLA) role have been widely used in many contexts [8-11].

Previous reviews have suggested that HRLAs may be effective in improving access to health services and addressing health inequalities, in part by providing cultural leverage $[12,13]$. Whilst evidence on cost-effectiveness is lacking, a perception exists that interventions involving trained but unqualified peer or lay advisers are cheaper than those involving health professionals. However, there are often hidden costs associated with coordination, training and supervision for these roles [14]. We therefore set out to examine systematically the evidence on the effectiveness of HRLAs and to determine in which areas they are likely to be cost-effective. Specifically, we were interested in HLRAs working in the developed world, where contexts differ significantly from those facing HRLAs in developing countries and where modifiable lifestyle behaviours pose a greater threat to health, as noted above. The results reported here are part of a larger evidence synthesis commissioned by the National Institute for Health Research (NIHR) Health Technology Assessment (HTA) Programme, which considered both qualitative and quantitative evidence for the effectiveness, equity and acceptability of different types of HRLA [15].

\section{Methods}

\section{Literature search}

Full details of the search for Medline are provided in Additional file 1 and details of strategies for the other databases are available from the full report [15]. The literature searches included a combination of HRLA, health improvement and methodological terms. The PICOS (Population, Interventions, Comparators, Outcomes and Study Designs) framework was used to generate search terms. Studies were included if they described interventions that were: delivered in developed countries by trained but generally unqualified HRLAs (paid workers or volunteers); consisted of education, training, support or counselling aimed at individuals or groups of peers with the explicit aim of health improvement; and were iterative (rather than a one-off event) and delivered in person, by telephone, post, online or electronically. Studies were excluded if they described (rather than evaluated) an intervention, related to acute care or a single episode of advicegiving, did not have the explicit aim of health improvement, or were not published in English. We also excluded studies focusing solely on advice or training delivered to children or adolescents, as there is already an established literature on peer education for young people. We included all studies with quantitative data on relevant interventions rather than restrict the review to full economic evaluations.

Articles published up to and including September 2008 were searched in the following bibliographic databases: ArticleFirst, ASSIA, British Humanities Index, Cinahl, DARE, Embase, Francis, IBSS, Medline, NHS Economic Evaluation Database, PAIS, Psycinfo, Science Citation Index, Social Science Citation Index, Social Services Abstracts, Sociological Abstracts, SIRS researcher, Web of Knowledge, Worldcat, Zetoc. The search string was modified in accordance with the functions available on each database. Grey literaturewas retrieved through searches using the Google search engine, with the first 100 results retrieved by each search strategy scanned for relevance; searches of specific websites; and contacting experts working in the field to request suggestions for relevant literature. Citation searches of the Science Citation Index and the Social Science Citation Index were carried out in order to identify all citations of those studies assessed as relevant. The reference lists of papers identified as relevant to the review were also checked.

Studies were assessed by two reviewers for their methodological rigour based on the Quality Assessment Tool for Quantitative Studies [16] and included in the review when they were rated as being of strong or moderate quality overall. Any disagreement between the reviewers was resolved through discussion to achieve consensus or, 
where required, review by a third member of the research team. Full details of the literature search are available in the published report [15].

\section{Data analysis and synthesis}

The disparate nature of the included studies precluded a meta-analysis of study findings. Instead, evidence on the effectiveness of HRLAs was assessed based on: i) the quality and nature of the studies; and ii) the target population. Where evidence existed on the cost-effectiveness of HRLA interventions we evaluated it. Generally this evidence was lacking and so we synthesised our own estimate of cost-effectiveness. We first grouped interventions according to the behaviour targeted as this dictated the size of the potential health gain. Within groups we then considered whether the reported interventions used similar or different modes of delivery. Typical modes of delivery included: community activities and mailshots (considered low intensity); telephone and group counselling (low to medium intensity); and personalised counselling (high intensity). Where studies in the same target behaviour group used the same mode of delivery, we selected one study to inform estimates of costs and effectiveness based on rigor of study design and relevance of the study population. Where interventions used a different mode of delivery, we compared them as alternative strategies, either against no intervention, or against non-HRLA delivered alternatives where these would be readily available. We ranked interventions according to effectiveness and eliminated dominated and extendedly dominated options prior to calculating incremental cost-effectiveness ratios (ICERs) [17]. The perspective of included studies was rarely stated, but none documented costs arising outside of the health sector. Where we synthesized cost-effectiveness estimates we considered only health benefits and costs falling on the health sector.

Our method built on previous work which examined the cost-effectiveness of interventions to incentivise physician activities which improve patients' health [18]. This study recognised that the total cost of such activities include the cost of the intervention and the impact on overall health costs of the change in patient health. Hence the traditional formula for calculating the ICER can be rewritten to express changes in costs as the sum of these two components. Few studies report long term health gains. However, estimates of the long term health gain from a change in underlying behaviour may be available. If we assume long term health gains are proportional to the magnitude or extent of behaviour change the health gain attributable to the intervention can be expressed as a product of the change in outcomes measured in patients and the health gain attributable to a unit change in outcome. The ICER can then be expressed as:

$$
\begin{aligned}
\mathrm{ICER} & =\Delta \mathrm{C} / \Delta \mathrm{Q}=\left(\Delta \mathrm{C}_{\mathrm{i}}+\Delta \mathrm{C}_{\mathrm{bh}}\right) / \Delta \mathrm{Q} \\
& =\left(\Delta \mathrm{C}_{\mathrm{i}}+\Delta \mathrm{C}_{\mathrm{bh}}\right) / \Delta \mathrm{Q}_{\mathrm{bh}} \cdot \mathrm{e}_{\mathrm{i}}
\end{aligned}
$$

where $\Delta \mathrm{C}$ is the change in overall (health) costs, $\Delta \mathrm{Q}$ is the change in outcome attributable to the intervention, $\Delta C_{i}$ is the cost of the intervention, $\Delta \mathrm{C}_{\mathrm{bh}}$ is the overall change in health care costs from the underlying behaviour change, $\Delta \mathrm{Q}_{\text {bh }}$ is the health gain attributable to a unit change in behaviour, and $e_{i}$ is the mean proportion or degree of change achieved by the intervention population.

We determined $e_{i}$ from the primary outcome reported in each study. These were typically changes in behaviour (such as mammography attendance) or in physiological markers (such as HbA1c concentration). We assessed assumptions underpinning analysis in each study and substituted conservative results where assumptions may have been generous (e.g. we assumed smoking cessation participants lost to follow up had resumed smoking). We searched the published literature for modelling studies reporting the lifetime health gains in Quality Adjusted Life Years [19] (QALYs) $\left(\Delta \mathrm{Q}_{\mathrm{bh}}\right)$ and health care cost impacts $\left(\Delta \mathrm{C}_{\mathrm{bh}}\right)$ attributable to changes in behaviour or associated with changes in physiological health markers. Where more than one modelling study was available we selected the study based on quality and applicability to a UK context.

Intervention costs $\left(\Delta \mathrm{C}_{\mathrm{i}}\right)$ were taken from cost data reported in the studies reviewed, where these were available. Costs in foreign currency were converted to UK pounds and inflated to 2008 prices. In the absence of cost data we estimated programme costs. We estimated the total staff time to deliver an intervention, and applied a unit cost which included training and overheads from an authoritative UK source (Unit Costs of Health and Social Care) for an occupation that most closely matched the activity described in the intervention [20]. Details of the costs estimations are given in Additional file 1.

Little evidence is available on long-term recidivism for lifestyle change interventions [21,22]. The exception to this is smoking cessation, where recidivism is substantial [23]. Seven year follow-up of participants in a lifestyle intervention for diabetes management showed that the majority had relapsed [24]. We assumed that interventions would be repeated in the third, sixth and tenth year after the initial intervention, and that despite this $50 \%$ of participants achieving change at follow-up would subsequently relapse gaining no health benefits. Costs for implementing the intervention in years three, six and ten were added to the implementation costs in the first year after discounting at $3.5 \%$ per annum to estimate the total cost of the intervention. We did not assume that smoking cessation interventions would be repeated; 
instead, we assumed that $75 \%$ of abstainers after three months would subsequently relapse based on data from 1990 California Tobacco Survey [25]. We did not assume that HIV prevention work amongst drug users would be repeated in subsequent years, given the transient nature of the population; instead, we applied conventional, conservative assumptions that intervention effects lasted for the duration of observation only.

Where estimated ICERs were likely to be sensitive to assumptions around health gains or recidivism, we tested these assumptions in sensitivity analysis.

\section{Results}

After quality appraisal 24 met the inclusion criteria for the review. Figure 1 illustrates the search results and screening process.
Interventions included in the review were grouped into eight health areas: general chronic disease management; management of type 2 diabetes; promotion of smoking cessation; breast cancer screening; prevention of HIV infection; healthy diet and/or physical activity; breastfeeding; and mental health. Each of these areas is described in detail below.

\section{General chronic disease management}

Five studies were reviewed on the subject of lay-led disease management for a range of chronic conditions [26-30]. Three of these [26-28] were conducted in the UK, including an evaluation of the national Expert Patients Programme [26]. All of the studies reported significant improvements in patient self-efficacy and self care behaviour attributable to the intervention. Barlow

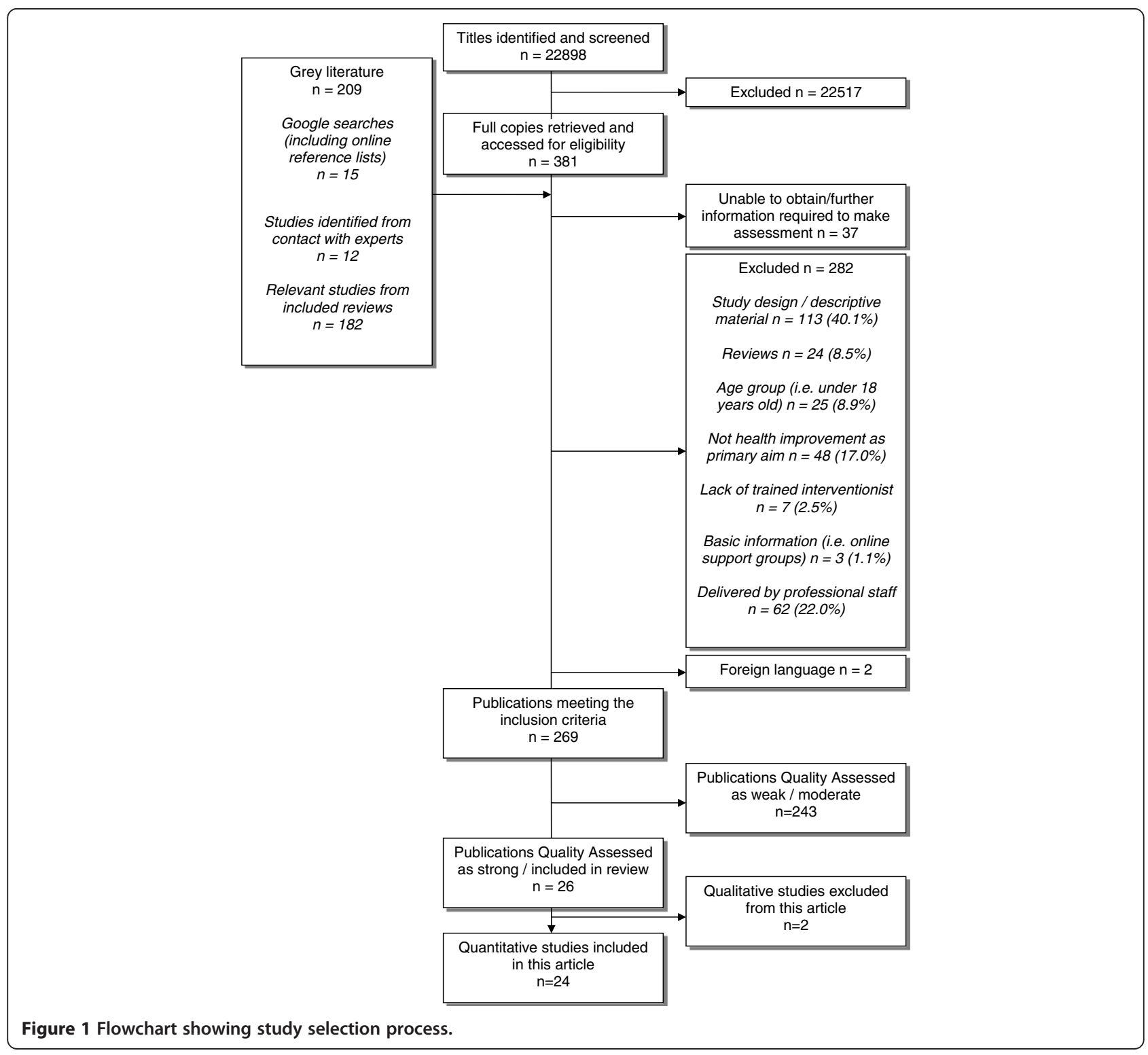


et al. [27] demonstrated statistically significant improvements in anxiety and depression. Kennedy et al. [26] reported improvements in physical health, Lorig et al. (2003) [29] found significant improvements in pain, and both studies reported improvements in psychological wellbeing and health distress. There was some evidence that health improvements were maintained over time. The extent to which improvements in self-efficacy and symptom management translate into gains in healthrelated quality of life (HRQoL) is unclear, although the impact of chronic diseases on HRQoL is likely to be considerable.

Evidence of the impact of interventions on health care utilisation was inconsistent. Kennedy et al. [26] reported reductions in both primary and secondary care attributable to the intervention. The differences were not statistically significant, but a reduction in inpatient days in the intervention arm was sufficient to offset the intervention cost. Griffiths et al. [28] and Barlow et al. [27] found no impact on health care utilisation attributable to the intervention. Lorig et al. (1999) [30] found a reduction in inpatient days but no reduction in primary care. Lorig et al. (2003) [29] found a reduction in primary care and emergency admissions, but no change in inpatient days.

\section{Estimating cost-effectiveness}

Richardson et al. undertook a cost-effectiveness analysis of the Expert Patients Programme, and reported a 94\% probability that it was cost-effective at a threshold of $£ 20,000$ per QALY [31]. This analysis appears to be robust, but may lack generalizability. Most of the intervention costs ( $£ 250$ per patient) were offset by reductions in inpatient days, attributable to a few resource-intensive patients. Evidence from the other studies of the impact on secondary care is mixed. Nevertheless, the chronic disease management programmes offer the possibility of enabling patient empowerment at modest or zero overall cost.

\section{Management of type 2 diabetes}

Three studies reported different methods of delivering lay-led lifestyle and disease management advice to poor, urban patients with diabetes [32-34]. Young et al. [32] describes a low intensity telephone counselling intervention, Gary et al. [33] reports a group counselling intervention delivered by promotoras (a term for lay health advisers used by Spanish-speaking communities) and Lujan et al. [34] reports an individual counselling intervention. We analysed each intervention as an alternative approach to promoting diabetes disease management in marginalised populations. Change in HbA1c concentration, a well-recognised physiological marker of diabetes control [35], was the primary outcome of each inter- vention. All three studies reported falls in HbA1c levels of similar magnitude attributable to the intervention: $0.25 \%$ (95\% CI 0.08 to 0.73 ) in Lujan et al.; $0.31 \%$ (95\% CI 0.11 to 0.52 ) in Young et al.; and $0.30 \%$ (CI -0.18 to 0.78 ) in Gary et al. The cost of the telephone-based intervention in Young et al. (£258 per participant) is reported separately [36]. We estimated costs per participant of $£ 757$ and $£ 988$ for Gary et al. and Lujan et al. respectively. On the basis of these costs the telephone counselling intervention dominates the other two (it is cheaper and more effective). However, differences in baseline $\mathrm{HbA} 1 \mathrm{c}$ between intervention and control in Lujan et al. may have diminished the observed treatment effect through regression to the mean. Consequently, we applied a favourable assumption that the treatment effect in Lujan et $a l$. is the fall in HbA1c in the treatment arm between baseline and follow-up (0.45\%).

\section{Estimating cost-effectiveness}

We assumed a linear relationship between changes in HbA1c concentration and the resulting impact on health gains and costs. A number of studies have estimated the health gain in QALYs from improved control of HbA1c [37-42]. We applied a value of 0.38 QALYs per $1 \%$ fall in HbA1c concentration taken from the Center for Outcomes Research (CORE) diabetes model [37]. Estimates of the costs saved from a fall in HbA1c levels in a UK setting were taken from the Diabforecaster model ( $£ 686$ 2008 GBP for a fall from $8.0 \%$ to $7.0 \%$ ) [38]. In the base case analysis, the ICER for the telephone counselling intervention in Young et al. was $£ 13,600$. The community health worker intervention in Gary et al. is dominated by the telephone counselling intervention. The ICER for the promotora intervention in Lujan et al. is $£ 94,500$.

In a sensitivity analysis the number of patients who relapse (and gain no health improvement) was varied between $25 \%$ and $75 \%$. This is mathematically equivalent to assuming a QALY gain from HbA1c control of plus or minus 50\% (0.19 and 0.57 QALYs). The resulting ICER for Young et al. varied from $£ 8,400$ to $£ 28,800$, and the ICER for Gary et al. varied from $£ 62,400$ to $£ 191,000$. Hence, the conclusion that a low intensity intervention for the management of type 2 diabetes is cost-effective at thresholds acceptable in the UKis robust to variation in health gains or recidivism.

An additional study estimated an ICER at $£ 43,400$ for the intervention described in Young et al. and concluded that the intervention was not cost-effective [36]. The analysis used data from the Centers for Disease Control type 2 diabetes model, in which US style intensive management of HbA1c levels generate considerable additional health care costs from a fall in HbA1c [39]. Under UK practice style, the estimated overall health care costs 
attributable to a fall in $\mathrm{HbA1c}$ levels are negative, which would greatly reduce the ICER for the intervention in Young 2005.

\section{Smoking cessation}

Four reviewed studies focused on advice or counselling regarding smoking cessation [43-46]. The evidence in May et al. [43] suggests that a 'buddy system' based on mutual support is not effective. The population in Emmons et al. were cancer survivors which limits the generalizability of the study [44]. The population in Woodruff et al. [45] appears representative of individual, ethnically-targeted smoking cessation services. Consequently, we considered this intervention, and assumed a three month quit rate of $17.4 \%$ based on the intentionto-treat analysis rather than the primary results (which ignored those lost to follow-up). We estimated the cost of the intervention at $£ 215$ per participant.

Estimates of the average QALY gain for cessation from smoking by Fiscella and Franks [47], and Cromwell [48] concur (1.98 and 1.97 QALYs respectively), although recent evidence [49] would suggest both are conservative. Health care costs related to smoking are considerable [50]. The impact of smoking cessation on health care costs unrelated to smoking is less well established, but these will inevitably rise with increased longevity and may outweigh the direct cost savings [51]. We applied a conservative assumption that the overall cost savings were zero. We assumed that $50 \%$ of three-month abstainers would have relapsed at one year and that $50 \%$ of one-year abstainers would subsequently relapse, with all those who relapse gaining no health benefits. Hence $25 \%$ of three-month quitters were assumed to have quit permanently, gaining 1.97 QALYs each.

\section{Estimating cost-effectiveness}

We compared the HRLA intervention, to reasonable alternatives available. We assumed that 3\% of smokers who wish to stop are successful without seeking professional support [52]. The success rate of brief advice in conjunction with Nicotine Replacement Therapy or Buproprion was estimated at 4\%, [53] with an estimated cost of $£ 47$ [54]. Pharmacy-based smoking cessation services were assumed to deliver a $5 \%$ quit rate at $£ 55$ per participant [55]. We assumed an annual success rate of $10 \%$ for smokers' clinics and costs per participant of $£ 350$ [55].

Cost-effectiveness calculations are presented in Table 1. The data indicate that smokers' clinics are more effective than individual counselling from HRLAs, although costs are similar. Smokers' clinics are cost-effective and the preferred option. However, this intensive group-based therapy may not appeal to many smokers. Tailoring services to smokers' choices is likely to boost motivation and resulting quit rates. Hence, HRLA interventions might be cost-effective as an alternative service. Compared with willpower alone, the ICER for smoking cessation advice delivered by HRLAs is $£ 3800$.

\section{Mammography promotion}

Four studies evaluated HRLA interventions to promote breast cancer screening (mammography) [56-59]. Three studies [56-58] targeted broadly similar populations of poor, rural women, whereas the fourth [59] targeted recent immigrants to the US. The likelihood of specific barriers to mammography in non-native language speaking communities limits the generalizability of the latter study. Hence we considered two types of intervention: a low intensity intervention focusing on community events and mass mailshots as described in Andersen et al. [56], and a high intensity intervention based primarily on individual counselling as described by Earp et al. [57] and Paskett et al. [58] The treatment effect (2.5\% uptake) and costs (\$34 per participant, £34 2008 GBP) reported for the community arm in Andersen et al. were assumed for the low intensity intervention, and the treatment effect (15.2\% uptake) and costs (\$730 per participant, £657 2008 GBP) reported in Paskett et al. for the high intensity intervention.

A number of authors have modelled the cost-effectiveness of mammography to determine the optimum screening frequency/age range [60-65]. Recent modelling studies have reported lifetime benefits of mammography in the range 0.0324-0.0386 QALYs for biennial or triennial screening [60-62]. We used the estimate of 0.0386

Table 1 Cost-effectiveness calculations for various smoking cessation options

\begin{tabular}{|c|c|c|c|c|c|c|}
\hline & Cost $(£)$ & Annual quit rate & QALY gain & Incremental gain (QALYs) & Incremental cost (£) & ICER \\
\hline Willpower alone & 0 & $3 \%$ & 0.0295 & - & - & - \\
\hline Brief advice & 47 & $4 \%$ & 0.0396 & 0.0099 & 47 & Extendedly dominated \\
\hline Pharmacy services & 55 & $5 \%$ & 0.0495 & 0.0099 & 8 & $£ 2,800$ \\
\hline Tailored HRLA counselling & 215 & $8.7 \%$ & 0.0857 & 0.0364 & 160 & $£ 4,400$ \\
\hline Smokers' clinics & 350 & $10 \%$ & 0.0985 & 0.0128 & 135 & $£ 10,500$ \\
\hline
\end{tabular}


QALYs from Rojnik et al. [60] which is generous, and matches UK screening policy (triennial from 50 to 70 years of age). Overall health care costs from mammography of $€ 191$ ( $£ 1482008$ GBP) were taken from the same study.

\section{Estimating cost-effectiveness}

The ICER for the low-intensity intervention was $£ 251,000$ and the ICER for the higher intensity intervention was $£ 896,000$. Sensitivity analysis assuming 0\% relapse without repeating the HRLA intervention generated an ICER for the low intensity intervention of $£ 39,000$. Andersen et al. [56] reports a cost-effectiveness analysis of the community activity intervention, giving a cost per life-year saved of US\$56,000 (1995 US\$) or $£ 56,000$ (2008 GBP). Description of the analysis is scant and no mention is made of any allowance for recidivism. Nevertheless, the analysis supports the conclusion that the HRLA intervention would not be considered costeffective in the UK.

\section{HIV prevention}

Two reviewed studies describe the training of illegal drug users to deliver HIV prevention messages, primarily promoting condom use and the sterilisation of drug injection equipment [66,67]. Whilst both studies are mainly qualitative, quantitative data on each intervention has also been published $[68,69]$. The two interventions are similar, but Dickson-Gomez et al. (2003) [66] included a control group who received an appropriate comparison intervention, and therefore analysis was based on that study. The study reports reduction in both risky sexual and injection behaviours. However, an ordinal scale was used to define the magnitude of risky behaviours obfuscating the absolute reduction following the intervention, and the authors elected to ignore any reported increases in risky behaviour which may have biased results.

\section{Estimating cost-effectiveness}

Data in Latkin et al. indicates the intervention was more effective in reducing needle-sharing than unsafe sex [68]. We considered only the impact of reductions in needlesharing given that the risk of infection from shared needles is higher than from unprotected vaginal sex (although not unprotected anal sex) [70]. We based estimates of infections avoided on a Bernoulli-process model of transmission, where probability of infection is a function of the number of unsafe acts, the risk of transmission from an unsafe act, and the general prevalence of disease (equation 1 below) [71]. We applied the standard assumptions that the observed reduction in risk behaviour occurs only for the duration of the intervention, and that persons prevented from infection are not subsequently infected.

$$
\begin{aligned}
& \text { Probability of infection P } \\
& \quad=1-\left\{(1-\pi)+\pi\left(1-\left(a_{d} \cdot a_{i}\right)\right)\right\}^{n}
\end{aligned}
$$

where $\pi=$ HIV prevalence

$\alpha_{d}=$ risk of infection of needle used by sero-positive user $=0.9$ [72].

$\alpha_{i}=$ risk of infection from infected needle $=0.0067$ [73]

$\mathrm{n}=$ number of incidences of needle sharing

We considered a London setting where increased HIV prevalence of 4\% [74] amongst drug users would render the programme more effective than in the rest of England (prevalence $0.6 \%$ [75]). We estimated 0.00722 cases of HIV infection avoided for the intervention and 0.00288 for the control, an incremental gain from the intervention of 0.004332 cases averted (details in Additional file 1).

We assumed that averting one case of HIV infection avoided $£ 143,000$ [76] and generated a health gain of around 5.37 QALYs [77]. On that basis, the intervention saves $£ 619$ and generates 0.0233 QALYs compared to the control, or $£ 1,032$ and 0.0388 QALYs compared to no intervention. Total intervention costs were estimated at $£ 59,200$. The resulting ICER for the HRLA programme is $(59,200-619) / 0.0233=£ 2,514,000$ per QALY compared to the control, and $(59,200-1032) / 0.0388=£ 1,499,000$ compared to no intervention.

\section{Diet and physical activity}

Five studies evaluated an intervention aiming to improve diet and exercise levels in a marginalised community using culturally tailored approaches [78-82]. Keyserling et al. [78] described an intervention for women with diabetes, while the four other studies [79-82] were aimed at healthy adults. Each intervention involved community health workers or promotoras delivering individual counselling and advice with the goal of increasing exercise and/or improving diets.

The evidence of improvements in diet, based on selfreported intake, is undermined by evidence of underreporting of consumption. There is evidence of improvement in fat intake and fruit and vegetable consumption, but studies measuring weight showed no change. Physiological evidence reported in Staten et al. is mixed [79]. Keyserling et al. reports no improvements in HbA1c levels despite extensive health advice and counselling [78]. The evidence of unreliability of self-reported dietary intake might also lead us to doubt the self-reported evidence of increased physical activity. Elder 2006 reports that improvements from the intervention observed at three months dissipated at six and 12-month follow-up [80]. 
Overall, the physiological data conflict with the selfreported improvements in diet or exercise, and there is evidence that any possible changes are not sustained. These results suggest that individual counselling from HRLAs is ineffective at achieving long-term changes in diet or exercise patterns. Consequently, these interventions do not appear to be cost-effective.

\section{Breastfeeding}

Two studies concerned breastfeeding: the study by Dennis et al. [83] took place in Canada in circumstances similar to a UK context, while the study by Morrow et al. [84] was conducted in Mexico. The Canadian intervention utilised telephone support from a woman experienced with breastfeeding, in addition to standard practice. The study reported risk ratios at four, eight and 12 weeks for increased breastfeeding attributable to the programme. The risk ratio at six weeks was estimated at 1.12 (95\% confidence interval: $1.01-2.00)$ using regression and combined with UK data on breastfeeding [85] to estimate the peer support programme could increase breastfeeding at six weeks in the UK from $48 \%$ to $54 \%$.

Reported benefits of breastfeeding include gains in Intelligence Quotient [86] and reductions in obesity [87] and childhood type 1 diabetes [88]. We did not, however, find any studies estimating a gain in QALYs from breastfeeding. Consequently, we could not estimate an ICER for the HRLA intervention described in Dennis et al.

\section{Mental health promotion}

Only one study was identified that addressed mental health issues [89]. The intervention involved an experienced parent providing support to families of children with selected chronic diseases. The control group were given a telephone number through which they could access the HRLA, but only two mothers called the number, suggesting selective demoralisation occurred in the control arm.

The study reported a statistically significant improvement in anxiety levels of 2.1 points on the Psychiatric Symptom Index (range 0 to 100) [90] attributable to the intervention. Costs were not reported but the intervention consumed ten hours of contact time plus telephone support from paid peer advisers, in addition to support from a clinical specialist. Hence costs were likely to be large. We found insufficient evidence in the literature to attach a QALY gain to reductions in anxiety. Hence, we did not calculate an ICER. The change in anxiety appears to be modest and the comparator used in the trial may have biased this estimate. Given the resource intensive nature of the intervention it is unlikely to be costeffective.

\section{Discussion}

\section{Main findings of this study}

Our analysis suggests that peer- or lay-delivered smoking cessation interventions are highly cost-effective compared to no intervention. Whilst smokers' clinics are more cost-effective they may not be accessed by marginalised groups. Promotion of uptake of mammography, exercise and healthy eating, or HIV prevention activities by HRLAs are not cost-effective. Programmes directed towards improved chronic disease management have the potential to be cost-effective, particularly for patients with type 2 diabetes. The conclusions on physical activity and healthy eating flow from a lack of evidence of effectiveness in these areas. Where there is evidence of effectiveness, lay health advisers are not always costeffective. The key driver is the size of the potential health gain from the behaviour promoted. This is large for smoking cessation, and justifies a relatively intensive intervention. The gain from mammography, recently estimated to be an additional 9.2 days life expectancy [91], is simply insufficient to justify even a low intensity promotion programme. The benefits from improved management of diabetes are potentially large, and may justify a low intensity intervention. Whilst the benefit of averting HIV infection is large, the health gain from reducing needle-sharing is modest, and consequently HIV risk reduction programmes are unlikely to be costeffective in the UK. We were unable to link evidence of effectiveness of HRLA interventions in mental health and breastfeeding promotion with changes in HRQoL.

\section{Strengths and limitations of this study}

This analysis is based on the first systematic review of the diverse range of HRLA roles to be found in developed countries [15]. The review was comprehensive both in its scope and in the focus on acceptability, effectiveness and cost-effectiveness of interventions. Nevertheless, we found few studies assessing outcomes after one year. Restrictions on inclusion by study design and quality assessment excluded many weaker studies. The remaining studies were predominantly single focus interventions, with defined and often standardised protocols, and a predominant focus on individual behaviour change rather than community development. The small number of studies reviewed in each area raises the possibility of publication bias leading to an over-estimate of the effectiveness of HRLA programmes. We lacked sufficient evidence to determine whether any of the interventions reduced or widened health inequalities. However, effective interventions targeted at marginalised communities are likely to reduce the socio-economic gradient in unhealthy behaviours.

The benefits of lifestyle interventions are typically accrued decades into the future for younger people, hence 
capturing long term outcomes is challenging. Consequently, we had to model long term impact from surrogate short term outcomes which raises concerns about whether the observed behaviour changes were maintained. In the absence of evidence we applied conservative assumptions on the maintenance of behaviour changes rather than simply assuming that no relapse occurred. Nevertheless, our conclusions on mammography promotion were robust to the very generous assumption, commonly applied, that increased adherence is maintained for life. Data on long term maintenance would greatly improve the quality of evaluations of health promotion programmes. We also selected conservative estimates of the health gain from lifestyle changes with regard to our resulting inference - high estimates for mammography and low estimates for smoking cessation - and we explored the impact of changing assumptions with sensitivity analysis. Inferences around mammography promotion and management of type 2 diabetes were robust to this analysis.

\section{Interpretation of findings and relationship to existing knowledge}

Few studies have evaluated the cost-effectiveness of HRLA interventions. Of the three studies we found which did, $[31,36,56]$ two had methodological weaknesses $[36,56]$. Andersen et al. appear to make the commonly applied but unrealistic assumption that behaviour change observed during follow-up is maintained for life [56]. In contrast, assumptions of cost increases from improved management of type 2 diabetes made by Mason et al. in the evaluation of the telephone based counselling intervention appear unrealistic [36]. We drew on the approach of Mason et al., but sought to apply the most appropriate estimates of the costs and effects of behaviour changes along with more realistic assumptions on adherence. With the exception of smoking cessation, there was very little evidence to guide assumptions on adherence and so we tested our assumptions in sensitivity analysis. This study is the first to evaluate the cost-effectiveness of HRLA interventions across a diverse range of health behaviours using the same methodology and applying conservative assumptions on adherence. The results indicate that HRLAs can be cost-effective when they target behaviours associated with significant detriments to health.

\section{Conclusion and implications}

Cost-effectiveness of HRLAs are strongly influenced by the size of the health gain from the lifestyle changes targeted. For example, lifetime adherence to mammography screening yields health gains of around 0.04 QALYs compared to 0.4 QALYs for a person with type 2 diabetes reducing their glycosylated haemoglobin by $1 \%$, and 2.0 QALYs for smoking cessation. Consequently the potential health gains from interventions that promote mammography are small compared to those promoting smoking cessation. HRLAs are likely to be most profitably employed delivering interventions with the potential to generate large health gains. They are unlikely to be cost-effective when targeting behaviours with only very modest potential to increase expected HRQoL (such as attendance at mammographic screening). Further research on adherence to short term behaviour changes might facilitate more informed assumptions on the long term maintenance of behaviour changes which underpin estimates of the cost-effectiveness of interventions aimed at improving unhealthy lifestyles.

\section{Additional file}

Additional file 1: Estimated costs and calculations of cost-

effectivenesss of interventions.

\section{Abbreviations}

HRLA: Health related lifestyle adviser; QALY: Quality adjusted life year; ICER: Incremental cost-effectiveness ratio; HRQoL: Health related quality of life; HIV: Human immunodeficiency virus.

\section{Competing interests}

The authors declare that they have no competing interests.

\section{Authors' contributions}

SC and MW conceived the study; CD and MP designed the study economics aspects; SC, ML, NF, KD, LG and SV undertook the systematic review; MP undertook the analysis; MP, SV and SC wrote the manuscript; MW, CD, KD, NF and ML commented on and revised drafts; SC is guarantor. All authors read and approved the final manuscript.

\section{Authors' information}

Cost-effectiveness and Resource Allocation (IF=1.68) - Instructions for authors: http://www.resource-allocation.com/authors/instructions/research.

\section{Acknowledgments}

The authors wish to thank all of those involved in the project advisory group, all contributors to the research (Lesley Geddes, Natalie Forster, Susan Michie, Philip Hodgson) and also the administrative support staff at Northumbria University. This study is based on an evidence synthesis funded by the NIHR Health Technology Assessment Programme (project number 07/26/03). The views and opinions expressed therein are those of the authors and do not necessarily reflect those of the Department of Health.

At the time of conducting the study, SC, CD and MW were funded in part as members of Fuse (the Centre for Translational Research in Public Health), a UK Clinical Research Collaboration (UKCRC) Public Health Research Centre of Excellence. Funding from the British Heart Foundation, Cancer Research UK, Economic and Social Research Council, Medical Research Council, and the National Institute for Health Research (NIHR) under the auspices of UKCRC, is gratefully acknowledged.

SV was funded by an NIHR doctoral research fellowship from September 2008 to September 2011 (project number RTF/08/01/096).

\section{Author details}

${ }^{1}$ Health Economics, London School of Hygiene \& Tropical Medicine, 15-17 Tavistock Place, London WC1H 9SH, UK. ${ }^{2}$ Centre for Public Policy and Health (CPPH), School of Medicine, Pharmacy and Health, Wolfson Research Institute for Health and Wellbeing, Durham University Queen's Campus,

Stockton-on-Tees TS17 6BH, UK. ${ }^{3}$ Yunus Centre for Social Business \& Health, Glasgow Caledonian University, Level 3 - Buchanan House, 58 Port Dundas Road, Glasgow G4 OBA, UK. ${ }^{4}$ Institute of Health \& Society/Fuse UKCRC Centre 
for Translational Research in Public Health, Newcastle University, Baddiley-Clark Building, Faculty of Medical Sciences, Newcastle University, Newcastle upon Tyne NE2 4AX, UK. ${ }^{5}$ Faculty of Health \& Life Sciences/Fuse UKCRC Centre for Translational Research in Public Health, Room H012, Coach Lane Campus East, Northumbria University, Newcastle-upon-Tyne NE7 7XA, UK. ${ }^{6}$ School of Nursing Sciences, Faculty of Medicine and Health Sciences, Edith Cavell Building, University of East Anglia, Norwich NR4 7TJ, UK.

Received: 26 April 2013 Accepted: 28 September 2013

Published: 4 December 2013

\section{References}

1. World Health Organization: The World Health Report 2002 - Reducing risks, promoting healthy life. Geneva: World Health Organization; 2002.

2. Department of Health: Tackling Health Inequalities: a programme for action. London: Department of Health; 2003.

3. Liao Y, Tucker P, Okoro CA, Giles WH, Mokdad AH, Harris VB: Reach 2010 surveillance for health status in minority communities - United States, 2001-2001. MMWR Surveill Summ 2004, 53(6):1-36.

4. Buck D, Frosini F: Clustering of unhealthy behaviours over time. Implications for policy and practice. London: King's Fund; 2012. Available at http://www. kingsfund.org.uk/publications/clustering-unhealthy-behaviours-over-time.

5. Department of Health: Choosing health: making healthy choices easier. London: The Stationery Office; 2004

6. Kangas l: "Lay' and 'expert': illness knowledge constructions in the sociology of health and illness'. Health 2002, 6(3):301-304.

7. Lorig K: Partnerships between expert patients and physicians. Lancet 2002, 359:814-815.

8. Visram S, Drinkwater C: Health Trainers: a review of the evidence. Newcastle-upon-Tyne: Northumbria University; 2005.

9. Visram S, Geddes L, Carr SM, Drinkwater C: An Evaluation of the Early Adopter Phase of the Health Trainers Project in the North East. Newcastle-upon-Tyne: Northumbria University; 2006.

10. World Health Organization: Strengthening the performance of community health workers in primany care: report of a WHO Study Group. Geneva: WHO; 1989.

11. Lewin SA, Dick P, Pond P, et al: Lay health workers in primary and community health care. Cochrane Database Syst Rev 2005, Issue 2: Art. no. CD004015.

12. Webel AR, Okonsky J, Trompeta J, Holzema WL: A systematic review of the effectiveness of peer-based interventions on health-related behaviours in adults. Am J Public Health 2010, 100:247-253.

13. Viswanathan M, Kraschnewski J, Nishikawa B, Morgan LC, Thieda P, Honeycutt A, Lohr KN, Jonas D: Outcomes of Community Health Worker Interventions. US: Agency for Healthcare Research and Quality; 2009. Evidence Reports/Technology Assessments, No. 181. Report No. 09-E014.

14. Buchan J, DalPoz M: 'Skill mix in the health care workforce: reviewing the evidence'. Bull World Health Org 2002, 80(7):575-580

15. Carr SM, Lhussier M, Forster N, Geddes L, Deane K, Pennington M, Visram S, White M, Michie S, Donaldson C, Hildreth A: An evidence synthesis of qualitative and quantitative research on component intervention techniques, effectiveness, cost effectiveness, equity and acceptability of different versions of health-related lifestyle adviser role in improving health. Health Technol Assess 2011, 15(9).

16. Effective Public Health Practice Project: Quality Assessment Tool for Quantitative Studies. Ontario, Canada: Public Health Research, Education and Development Program (PHRED); 2003

17. Karlsson $\mathrm{G}$, Johannesson $\mathrm{M}$ : The decision rules of cost-effectiveness analysis. Pharmacoeconomics 1996, 9:113-120.

18. Mason J, Freemantle N, Nazareth I, Eccles M, Haines A, Drummond M: When is it cost-effective to change the behavior of health professionals? JAMA 2001, 286(23):2988-2992.

19. Loomes G, McKenzie L: The use of QALYs in health care decision making. Soc Sci Med 1989, 28:299-308.

20. Curtis L: Unit costs of health and social care. Kent: Personal Social Services Research Unit; 2008.

21. Anderson JW, Konz EC, Frederich RC, Wood CL: Long-term weight-loss maintenance: a meta-analysis of US studies. Am J Clin Nutr 2001, 74:579-584.

22. Orleans $\mathrm{CT}$ : Promoting the maintenance of health behavior change: recommendations for the next generation of research and practice. Health Psychol 2000, 19(1, suppl):76-83.
23. Hughes $J R$, Peters EN, Naud S: Relapse to smoking after 1 year of abstinence: a meta-analysis. Addict Behav 2008, 33:1516-1520.

24. Toobert DJ, Stryker LA, Barrera M, Glasgow RE: Seven-year follow-up of a multiple-health behavior diabetes intervention - HMC. Am J Health Behav 2010, 34(6):680-694.

25. Gilpin EA, Pierce JP, Farkas AJ: Duration of smoking abstinence and success in quitting. J Natl Cancer Inst 1997, 89(8):572-576.

26. Kennedy A: The effectiveness and cost effectiveness of a national lay-led self care support programme for patients with long-term conditions: a pragmatic randomised controlled trial. J Epidemiol Community Health 2007, 61:254-261.

27. Barlow JH, Turner AP, Wright CC: A randomized controlled study of the arthritis management programme in the UK. Health Educ Res 2000 15:665-680

28. Griffiths C, Motlib J, Azad A, Ramsay J, Eldridge S, Feder G, et al: Randomised controlled trial of a lay-led self-management programme for Bangladeshi patients with chronic disease. Br J Gen Pract 2005, 55:831-837.

29. Lorig KR, Ritter PL, Gonzalez VM: Hispanic chronic disease self management. Nursing Res 2003, 52:361-369.

30. Lorig KR, Sobel DS, Stewart AL, Brown BW, Bandhura A, Ritter P, et al: Evidence suggesting that a chronic disease self-management program can improve health status while reducing hospitalization. Med Care 1999, 37:5-14.

31. Richardson G, Kennedy A, Reeves D, Bower P, Lee V, Middleton E, et al Cost effectiveness of the Expert Patients Programme (EPP) for patients with chronic conditions. J Epidemiol Community Health 2002, 62:361-367.

32. Young RJ: Proactive call centre treatment support (PACCTS) to improve glucose control in type 2 diabetes. Diabetes Care 2005, 28:278-282.

33. Gary TL: Randomized controlled trial of the effects of nurse case manager and community health worker interventions on risk factors for diabetes-related complications in urban African Americans. Prev Med 2003, 37:23-32.

34. Lujan J, Ostwald SK, Ortiz M: Promotora diabetes intervention for Mexican Americans. Diabetes Educ 2007, 33:660-670.

35. Stratton IM, Adler Al, Neil HA, Matthews DR, Manley SE, Cull CA, et al: Association of glycaemia with macrovascular and microvascular complications of type 2 diabetes (UKPDS 35): prospective observational study. BMJ 2000, 321:405-412.

36. Mason LM, Young RJ, New JP, Gibson JM, Long AF, Gambling T, et al: Economic analysis of a telemedicine intervention to improve glycemic control in patients with diabetes mellitus: illustration of a novel analytic model. Dis Manage Health Outcomes 2006, 14:377-385.

37. Valentine WJ, Palmer AJ, Nicklasson L, Cobden D, Roze S: Improving life expectancy and decreasing the incidence of complications associated with type 2 diabetes: a modelling study of $\mathrm{HbA1c}$ targets. Int $J$ Clin Pract 2006, 60:1138-1145.

38. McEwan P, Peters JR, Bergenheim K, Currie CJ: Evaluation of the costs and outcomes from changes in risk factors in type 2 diabetes using the Cardiff stochastic simulation cost-utility model (DiabForecaster). Curr Med Res Opin 2006, 22:121-129.

39. Group CDC Diabetes Cost-effectiveness Group: Cost-effectiveness of intensive glycemic control, intensified hypertension control, and serum cholesterol level reduction for type 2 diabetes. JAMA 2002, 287:2542-2551.

40. Bagust A, Hopkinson PK, Maier W, Currie CJ: An economic model of the long-term health care burden of type II diabetes. Diabetologia 2001, 44:2140-2155

41. Clarke PM, Gray AM, Briggs A, Farmer AJ, Fenn P, Stevens RJ, et al: A model to estimate the lifetime health outcomes of patients with type 2 diabetes: the United Kingdom Prospective Diabetes Study (UKPDS) outcomes model (UKPDS no. 68). Diabetologia 2004, 47:1747-1759

42. Brown JB, Russell A, Chan W, Pedula K, Aickin M: The global diabetes model: user friendly ersion 3.0. Diabetes Res Clin Pract 2000, 50(Suppl. 3):15-46.

43. May S, West R, Hajek P, McEwen A, McRobbie H: Randomised controlled trial of a social support ('buddy') intervention for smoking cessation. Patient Educ Couns 2006, 64:235-241. 
44. Emmons KM, Puelo E, Park E, Gritz ER, Butterfield RM, Weeks JC, et al: Peer-delivered smoking counseling for childhood cancer survivors increases rate of cessation: the partnership for health study. J Clin Oncol 2005, 23:6516-6523.

45. Woodruff SI: Evaluation of a culturally appropriate smoking cessation intervention for Latinos. Tob Control 2002, 11:361-367.

46. West $R$, Edwards M, Hajek P: A randomized controlled trial of a 'buddy' system to improve success at giving up smoking in general practice. Addiction 1998, 93:1007-1011.

47. Fiscella K, Franks P: Cost-effectiveness of the transdermal nicotine patch as an adjunct to physicians' smoking cessation counseling. JAMA 1996, 275:1247-1251.

48. Cromwell J, Bartosch WJ, Fiore MC, Hasselblad V, Baker T: Cost-effectiveness of the clinical practice recommendations in the AHCPR guideline for smoking cessation. JAMA 1997, 278:1759-1766.

49. Doll R, Peto R, Boreham J, Sutherland I: Mortality in relation to smoking: 50 Years' observations on male British doctors. BMJ 2004 328:1519-1528.

50. Rasmussen SR, Prescott E, Sorensen TIA, Sogaard J: The total lifetime health cost savings of smoking cessation to society. Eur J Public Health 2005, 15:601-606.

51. Barendregt JJ, Bonneux L, van der Maas PJ: The health care costs of smoking. N Engl J Med 1997, 337:1052-1057.

52. West R: Getting serious about stopping smoking. A review of products, services and techniques. London: No Smoking Day; 1997.

53. Woolacott NF, Jones L, Forbes CA, Mather LC, Sowden AJ, Song FJ, et al: The clinical effectiveness and cost-effectiveness of bupropion and nicotine replacement therapy for smoking cessation: a systematic review and economic evaluation. Health Technol Assess 2002, 6(16).

54. Stapleton JA, Lowin A, Russell MA: Prescription of transdermal nicotine patches for smoking cessation in general practice: evaluation of cost-effectiveness. Lancet 1999, 354:210-215.

55. Boyd KA, Briggs AH: Cost-effectiveness of pharmacy and group behavioural support smoking cessation services in Glasgow. Addiction 2009, 104:317-325.

56. Andersen MR, Hager M, Su M, Urban N: Analysis of the cost-effectiveness of mammography promotion by volunteers in rural communities. Health Educ Behav 2002, 29:755-770.

57. Earp JA, Eng E, O'Malley MS, Altpeter M, Rauscher G, Mayne L, et al: Increasing use of mammography among older, rural African American women: results from a community trial. Am J Public Health 2002, 92:646-654.

58. Paskett E, Tatum C, Rushing J, Michielutte R, Bell R, Foley $\mathrm{KL}$, et al: Randomised trial of an intervention to improve mammography utilisation among a tri-racial rural population of women. $J$ Nat Cancer Inst 2006, 98:1226-1237.

59. Bird JA, McPhee SJ, Ha NT, Le B, Davis T, Jenkins CNH: Opening pathways to cancer screening for Vietnamese-American women: lay health workers hold a key. Prev Med 1998, 27:821-829.

60. Rojnik K, Naversnik K, Mateovic-Rojnik T, Primiczakelj M: Probabilistic cost-effectiveness modeling of different breast cancer screening policies in Slovenia. Value Health 2008, 11:139-148.

61. Ahern $\mathrm{CH}$, Shen $\mathrm{Y}$ : Cost-effectiveness analysis of mammography and clinical breast examination strategies: a comparison with current guidelines. Cancer Epidemiol Biomarkers Prev 2009, 18:718-725.

62. Salzmann P, Kerlikowske K, Phillips K: Cost-effectiveness of extending screening mammography guidelines to include women 40 to 49 years of age. [Erratum appears in Ann Intern Med 1998;128:878]. Ann Int Med 1997, 127:955-965.

63. Stout NK, Rosenberg MA, Trentham-Dietz A, Smith MA, Robinson SM, Fryback DG: Retrospective cost-effectiveness analysis of screening mammography. J Nat Cancer Inst 2006, 98:774-782

64. Boer $\mathrm{R}$, de Koning $\mathrm{H}$, Threlfall A, Warmerdam $\mathrm{P}$, Street A, Friedman E, et al: Cost effectiveness of shortening screening interval or extending age range of NHS breast screening programme: computer simulation study. BMJ 1998, 317:376-379.

65. Szeto KL, Devlin NJ: The cost-effectiveness of mammography screening: evidence from a microsimulation model for New Zealand. Health Policy 1996, 38:101-115.

66. Dickson-Gomez J, Knowlton A, Latkin C: Hoppers and oldheads: qualitative evaluation of a volunteer AIDS outreach intervention. AIDS Behav 2003, 7:303-315.
67. Dickson-Gomez J, Weeks M, Martinez M, et al: Times and places: process evaluation of a peer led HIV prevention intervention. Subst Use Misuse 2006, 41:669-690.

68. Latkin CA, Sherman S, Knowlton A: HIV prevention among drug users: outcome of a network-oriented peer outreach intervention. Health Psychol 2003, 22:332-339.

69. Weeks MR, Dickson-Gomez J, Mosack KE, Convey M, Martinez M, Clair S: The risk avoidance partnership: training active drug users as peer health advocates. J Drug Issues 2006, 36:541-570.

70. Cohen DA, Wu SY, Farley TA: Comparing the cost-effectiveness of HIV prevention interventions. J Acquir Immune Defic Syndr 2004, 37:1404-1414.

71. Pinkerton SD, Abramson PR: The Bernoulli-process model of HIV transmission. In Handbook of economic evaluation of HIV prevention programmes. Edited by Holtgrave DR. New York, NY: Plenum Press; 1998.

72. Allard R: A mathematical model to describe the risk of infection from sharing injection equipment. J Acquir Immune Defic Syndr 1990, 3:1010-1016 [Erratum appears in J Acquir Immune Defic Syndr 1991;4:450]

73. Kaplan EH, Heimer R: A model-based estimate of HIV infectivity via needle sharing. J Acquir Immune Defic Syndr 1992, 5:1116-1118.

74. Health Protection Agency (HPA): HIV in the United Kingdom: 2008 report. London: Health Protection Agency, HIV and Sexually Transmitted Infections Department; 2008

75. Health Protection Agency (HPA): Unlinked Anonymous Prevalence Monitoring Programme: data to the end of 2007, IDUs survey. London: Health Protection Agency Centre for Infections; 2008.

76. Levy AR, James D, Johnston KM, Hogg RS, Harrigan PR, Harrigan BP, et al: The direct costs of HIV/AIDS care. Lancet Infect Dis 2006, 6:171-177.

77. Paltiel AD, Weinstein MC, Kimmel AD, Seage GR III, Losina E, Zhang $H$, et al: Expanded screening for HIV in the United States: an analysis of cost-effectiveness. N Engl J Med 2005, 352:586-595.

78. Keyserling TC, Samuel-Hodge CD, Ammerman AS, Ainsworth BE, Henríquez-Roldán $C F$, et al: $A$ randomized trial of an intervention to improve self-care behaviors of African-American women with type 2 diabetes: impact of physical activity. Diabetes Care 2002, 25:1576-1183.

79. Staten LK, Gregory-Mercado KY, Ranger-Moore J, Will JC, Giuliano AR, Ford ES, et al: Provider counselling, health education and community health workers: the Arizona WISEWOMAN project. J Women's Health 2004, 13:547-556

80. Elder JP, Ayala GX, Campbell NR, Arredondo EM, Slymen DJ, Baquero, et al: Long term effects of a communication intervention for Spanish-Dominant Latinas. Am J Prev Med 2006, 31:159-166.

81. Resnicow K, Campbell MK, Carr C, McCarty F, Wang T, Periasamy S, et al: Body and soul: a dietary intervention conducted through African American churches. Am J Prev Med 2004, 27:97-105.

82. Anand SS, Davis DA, Ahmed R, Jacobs R, Xie C, Hill A, et al: A family-based intervention to promote healthy lifestyles in an aboriginal community in Canada. Can J Public Health 2007, 98:447-452.

83. Dennis CL, Hodnett E, Gallop R, Chalmers B: The effect of peer support on breast-feeding duration among primiparous women: a randomised controlled trial. Canad Med Assoc J 2002, 166:21-28.

84. Morrow AL, Lourdes Guerrerro M, Shults J, Calva JJ, Lutter C, Bravo J, et al: Efficacy of home based peer counselling to promote exclusive breastfeeding: a randomised control trial. Lancet 1999, 353:1226-1231.

85. Scientific Advisory Committee on Nutrition: Infant Feeding Survey 2005: a commentary on infant feeding practices in the UK. London: Scientific Advisory Committee on Nutrition; 2008.

86. Quinn PJ, O'Callaghan M, Williams GM, Najman JM, Andersen MJ, Bor W: The effect of breastfeeding on child development at 5 years: a cohort study. J Paediatr Child Health 2001, 37:465-469.

87. Gillman MW, Rifas-Shiman SL, Camargo CA Jr, Berkey CS, Frazier AL, Rockett $H R$, et al: Risk of overweight among adolescents who were breastfed as infants. JAMA 2001, 285:2461-2467.

88. Sadauskaite-Kuehne V, Ludvigsson J, Padaiga Z, Jasinskiene E, Samuelsson U: Longer breastfeeding is an independent protective factor against development of type 1 diabetes mellitus in childhood. Diabetes Metabol Res Rev 2004, 20:150-157.

89. Ireys HT, Chernoff R, DeVet KA, Young K: Maternal outcomes of a randomised controlled trial of a community-based support program for families of children with chronic illnesses. Arch Pediatr Adolesc Med 2001, 155:771-777. 
90. Okun A, Stein RE, Bauman LJ, Silver EJ: Content validity of the psychiatric symptom index, CES-depression scale, and state-trait anxiety inventory from the perspective of DSM-IV. Psychol Rep 1996, 79(3 Pt 1):1059-1069.

91. Pharaoh PDP, Sewell B, Fitzsimmons D, Bennett HS, Pashayan N:

Cost-effectiveness of the NHS breast screening programme: life table model. BMJ 2013, 346:f2618. doi: 10.1136/bmj.f2618.

doi:10.1186/1478-7547-11-30

Cite this article as: Pennington et al: Cost-effectiveness of health-related lifestyle advice delivered by peer or lay advisors: synthesis of evidence from a systematic review. Cost Effectiveness and Resource Allocation 2013 11:30.

\section{Submit your next manuscript to BioMed Central and take full advantage of:}

- Convenient online submission

- Thorough peer review

- No space constraints or color figure charges

- Immediate publication on acceptance

- Inclusion in PubMed, CAS, Scopus and Google Scholar

- Research which is freely available for redistribution 